\title{
The world of fractals
}

\section{Peter Nagy ${ }^{1}$, Peter Tasnádi ${ }^{2}$}

${ }^{1}$ GAMF Faculty, John von Neumann University, Hungary, ${ }^{2}$ Faculty of Science, Eötvös Loránd University, Hungary.

\begin{abstract}
Fractals are fascinating geometric structures of nature which appear in more and more field of science, ranging from heartbeat characterization through, cancer research, stock exchange trends, meteorology, and computer graphics, up to digital image processing. Fractals can be created by simple algorithms that can be manifested with also simple computer programs. The necessary programs are available in the literature and they are easy to understand and manage for first-year undergraduate students too. Therefore fractals are suitable to attract student interest and improve their attitudes toward learning physics. In this paper, a teaching material that is freely available on the internet is described. In addition to reading the article, we strongly recommend that the reader download and try out the teaching material, as this is the best way to get a real picture of its usability. (csodafizika.hu/fractals.zip) Our main goal was that students work independently and gain experience on fractals with applying simple algorithms and providing pictures of the weird formations of fractals. However, the material has been planned for engineering students, besides its versatility, it was focused on the connection between chaos and fractals. Our intention was to make our student's interest arise by the simple geometry of fractals, and from this, we try to lead them to the study of simple nonlinear chaotic mechanical systems helping them to understand deeper the Newtonian mechanics. Various versions (in Hungarian) of the material were used in secondary school teaching and in the postgraduate teacher training of ELTE so we believe it is suitable for almost anyone who would like to become familiar with fractals.
\end{abstract}

Keywords: fractals; downable interactive teaching material. 


\section{Introduction}

The discovery of fractals shook our approach to the geometric nature of the world to the bottom: we suddenly realised that the 'customary' regular forms featured in the former image of geometry are, in fact, only a negligibly tiny segment of the living and non-living material objects, while fractals grasp the genuine geometry and fundamental harmony of nature much deeper [Mandelbrot (1982)] Fractals provide not only the deeper understanding and simulation possibilities for the forms of nature, but revive the childish affection in us towards the miracles and wonder of the world. Since their discovery, fractals are used in more and more field, ranging from heart beat characterisation through investigating equilibrium, cancer research, stock exchange trends, meteorology, and computer graphics, up to digital image processing.

In this paper the teaching material can only be briefly described, so we strongly recommend that the reader download the zip file from our website [csodafizika], unzip it, look through the illustrations and try out computer simulations. The original material found on our website is much richer and show better our intention than this shortened version presented in this study. The teaching material has been made for didactic purposes and relies heavily on internet based materials, available to anybody.

\subsection{The use of the material}

This material fits the curriculum of an optional course "Concepts of Modern Physics" which was introduced at Neumann János University about a decade and a half ago. (Four major areas have been discussed: relativity, quantum theory, chaos theory and fractals, and statistical physics.) Each student can freely choose one area from the four for the oral exam (in agreement with the lecturer). In previous years, only about $10-15 \%$ of the students chose "chaos theory and fractals", whereas, after the interactive teaching material has become available this proportion increased to over $30 \%$. Besides this material, other interactive elearning materials joining closely this one are offered for the students e.g. "The use of dynamics solver", "Chaotic motions" etc. The chaotic attractors often show fractal structure so the two materials can foster each other. One of the most important goals of the introductory physics courses is the use of calculus in Newtonian mechanics. However, according to our experiences students are reluctantly using differential equations at this time. Therefore we encourage them to use computer programs (e.g. Dynamics Solver) to solve differential equations. We have experienced, that students who used the teaching material on fractals more easily overcame their aversion to solving equations of motion and they understood deeper the Newtonian mechanics too. 
Based on the experience of the course, we have developed several versions of the e-learning material presented here. In the autumn term of MSc engineering education of Neumann University a new course "Applied Physics" was launched which involves a slightly modified version of this material too. In the postgraduate teacher training of the Eötvös University, a Hungarian version of this material is used that focuses on the secondary school level teaching of fractals. [fiztan]

\section{The teaching material}

We have attempted to make students understood the essence of fractals and to present their significance in nature and in sciences through a number of examples. Our main goal was that students work independently and gain experience in fractals by applying simple algorithms and providing pictures of the weird formations of fractals. We have also tried to make the mathematics used as simple as possible. Therefore the exact mathematical background of fractals sometimes remains hidden. The material consists of four parts. In the first part, basic concepts concerning fractals are introduced through the detailed discussion of well-known examples. In the second part, simple real-life procedures are shown that lead to fractal formations. In the third and fourth parts, computer-simulated fractals are shown. Firstly some well-known algorithm is discussed and after the fractal dimension of some chaotic attractor is demonstrated.

\subsection{What is fractal?}

As a vivid example the well-known series of pictures taken with different magnification about the English seacoast is shown [fractalfoundation]. When looking at one or another picture, you are not able to decide whether you see the entire coastline or only a small detail of it, and hence, you do not have any reliable visual reference point to estimate the scale of the picture. The coastline is a so-called self-similar form. If you magnify a detail of a selfsimilar phenomenon or of a material object, it is indistinguishably similar to the whole, or any other detail, therefore, if something is self-similar, its scale can't be determined.

After all, it should be recognized that England is an extremely weird geometric form: its perimeter is infinite, while its area is finite. Such formations are called fractals. For the purposes of their characterisation fractal dimension $D_{0}$ is introduced which is an extension of the customary concept of dimension. Fractal dimension can be defined practically through measuring instructions. The fractal configuration embedded in a geometric space of dimension $\mathrm{d}$ is measured by covering it with 'cubes' of dimension $\mathrm{d}$ and size of $r$ (scale) then counting the number $N(r)$ of 'cubes' necessary for the cover (box-counting method), finally, the $\ln N(r)$ values are plotted as a function of $\ln (1 / r)$ where a line should be got the steepness 
of which is the fractal dimension of the formation $D_{0}$ The dimension $D_{0}$ of the fractal is the following: $D_{0}=\frac{\ln N(r)}{\ln \frac{1}{r}}$.

Based on the foregoing, you can state that fractals:

- are infinitely complex, complicated geometric configurations, which are self-similar in an exact or approximate (statistical) way across several orders of magnitude (range) scale,

- when any of their characteristics are plotted on a log-log scale (such as surface, volume or mass) as a function of their size, then they provide a line the steepness of which is the (typically fractional) fractal dimension.Fractals surround us everywhere, especially in nature. Among other, the mountains, the trees, the lightning bolts, leaves, fjords, snowflakes and clouds are all fractal forms View the [csodafizika] downloadable material, the video entitled fractals_in_nature.mp4.

\subsection{Fractals of daily life}

Fractals can be created even in other simple ways. Bakers (or anybody) keep on folding and stretching dough when kneading it, since the best mixing procedure is achieved by the recursive algorithm of stretching and folding, resulting in a typical fractal nature. Various colour plasticine rods can also be folded and stretched in a similar way to obtain spectacular patterns (Figure 1).
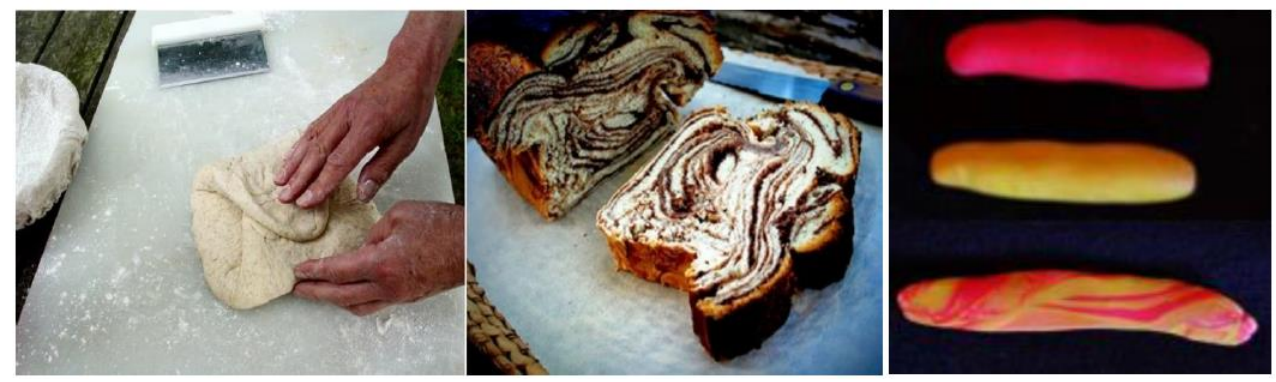

Figure 1: Fractal generation by stretching-bending algorithm (dough kneading and plasticine).

People are pleased when shaping wonderful fractal structures by their own hands, stirring paint pots or using the so-called marbling technique (see downloadable materials [csodafizika] marble.mp4, fractal_burning_art.mp4 and fractal_digital_art.mp4 videos). In spite of their irregularities, fractals have been incorporated into the arts quickly, giving birth to a branch of art working with novel means [authenticationinart.org]. 


\subsection{Deterministic fractals}

It follows from the self-similarity property that the simplest way of creating fractals is the application of recursive algorithms since in such cases generation takes place according to clear rules and the configurations obtained this way are called therefore deterministic fractals.

A practical and widely used way to specify and generate regular fractals with computers and recursive algorithms is the so-called L-system declarative language method. The method can be used easily with turtle graphics which provides an excellent opportunity to create several of well-known and spectacular fractal shapes.

As an example, it is worth taking the generation of the so-called Cantor-set fractal that is shown in Figure 2. The iterative steps are the following. From the internal part of a segment of the length of $L$ delete a segment of length $r L(r<1 / 2)$, and repeat this procedure with the remaining parts of the segments ad infinitum. (of course, the length of the deleted part is $r$ times of the length of the segment from which it is deleted).

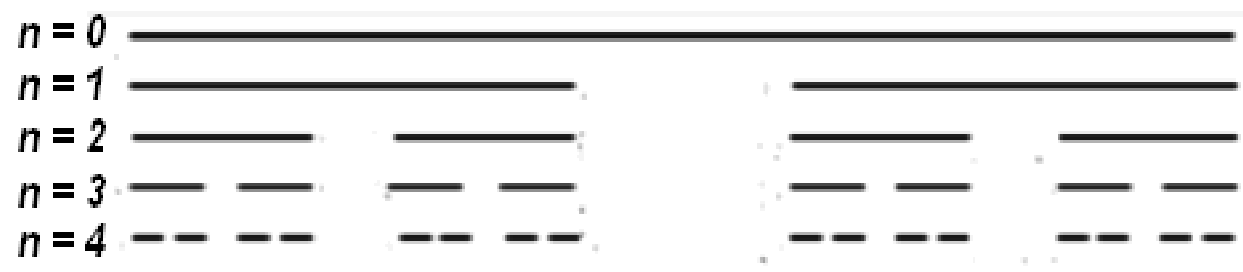

Figure 2: The first four steps of Cantor-set generation at $r=2 / 5$.

Based on the definition given above it can be easily realised that the fractal dimension of the Cantor-set is: $D_{0}=\frac{\ln 2}{\ln \left(\frac{1}{r}\right)}$ that is, for instance in the case seen in Figure $4 D_{0}=0.756$.

An important method of fractal generation is the 'projection' of fractals, that is their direct (Cartesian) product. In Figure 3 the first four steps of creating a Cantor-thread fractal are shown. In the horizontal direction, the Cantor-set recursion described earlier takes place, while perpendicular to it, in the vertical direction, it is continuous and therefore a linear (fibrous) structure is created. 


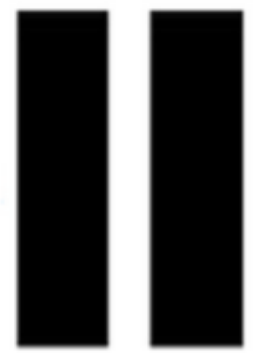

$n=1$

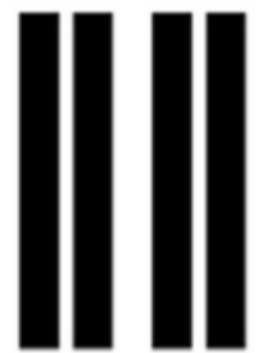

$n=2$

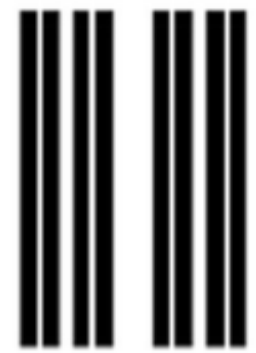

$n=3$

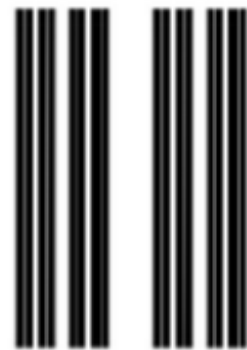

$n=4$

Figure 3: Cantor-thread fractal generation using direct product construction.

In Fig. 4 another well-known fractal, the Sierpinski-triangle (as well as the picture of a shell with similar patterns) can be seen. During the construction of the Sierpinski-triangle in each step, the central (white) triangles are deleted.

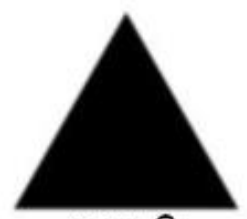

$n=0$

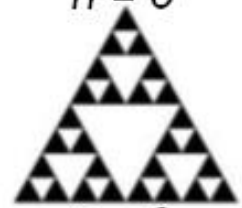

$n=3$

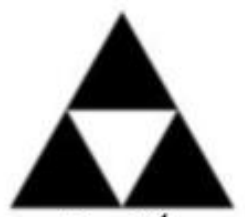

$n=1$

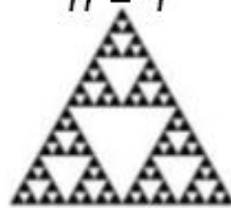

$n=4$

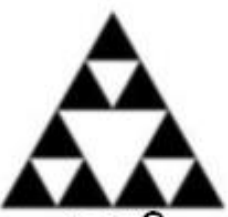

$n=2$

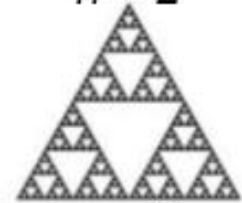

$n=5$

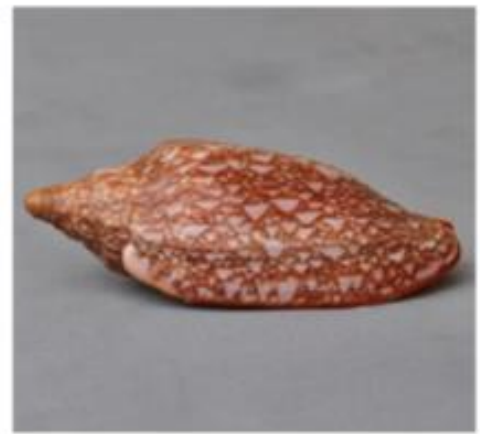

Figure 4: Generation of the Sierpinski-triangle and a shell with similar patterns.

\subsection{Stochastic fractals}

Take a so-called chaos game played using the random recursive algorithm below. 

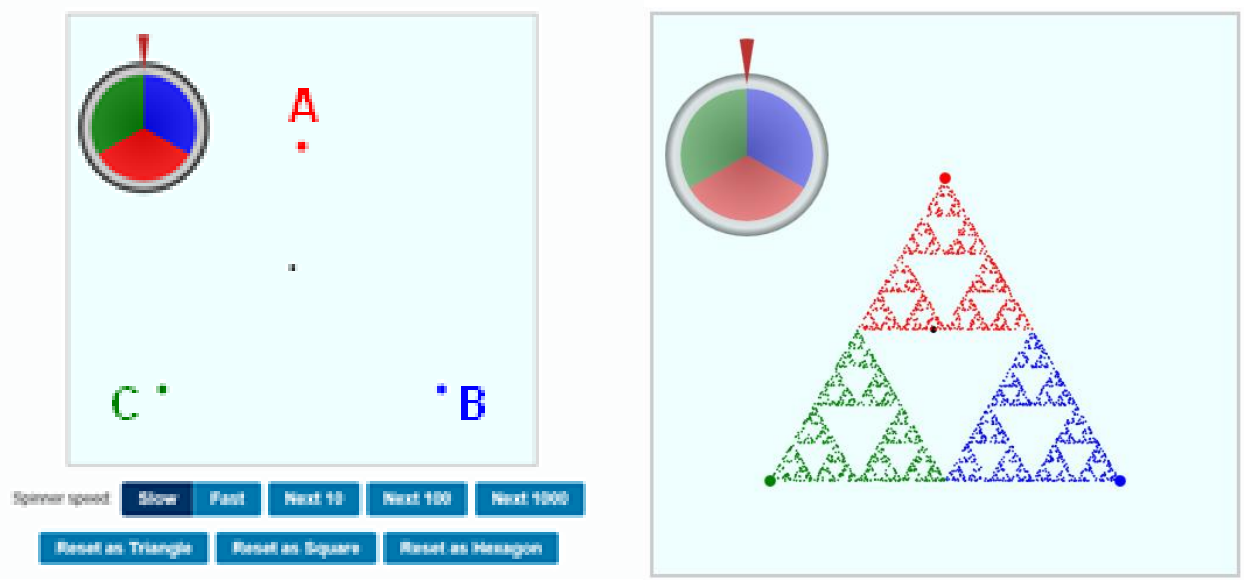

Figure 5: Screenshot of the computer aided implementation of the chaos game(left). The dots obtained in the course of the chaos game (right).

Select randomly a point in the inside of the (regular or nonregular) $\mathbf{A B C}$ triangle.

(*) Roll a dice.

Connect the selected point with

o corner $\mathbf{A}$, when the 1 or 2 comes out on the dice,

o corner $\mathbf{B}$ when the 3 or 4 comes out on the dice,

o corner $\mathbf{C}$, when the 5 or 6 comes out on the dice,

the new current point will be the bisecting point of the connecting segment, jump to $\left(^{*}\right)$.

The algorithm is found in the chaosgame folder of the downloadable materials [csodafizika], it is recommended to try it. (it can be started using the index.html_file).

It can be experienced with great surprise that the set of points obtained (Fig. 5) will be quite similar all the times to the well-known Sierpinski fractal (Figure 4), which is generated by a deterministic algorithm, while this game is based on a random (stochastic) algorithm. To put it more formally, the Sierpinski fractal contains with a probability of 1 the series of points obtained over any runs in the game, or, in the terms of chaos theory, the attractor to which the motion and colour of the current point belong is itself the Sierpinski-fractal.

\subsection{Chaos and Fractals}

There are several simple systems of low degrees of freedom the motion of which can be described using simple deterministic principles (in fact, by some non-linear dynamic equations) but their motion is complex and exhibit irregular (non-periodical, complicated) behavior [Nagy, Tasnádi (2014), Tél, Gruiz (2006)]. The motion is chaotic that is: 
- due to the extreme sensitivity to the initial conditions the errors in the initial state are exponentially growing, therefore the behaviour of the system is practically is unpredictable for a longer-term (thus only a probability description can be provided),

- in phase space, the long term behaviour of the system can be characterized by special geometric structure and order which often exhibit fractal structure.

Chaotic movement is ubiquitous in the world, so it often appears in our environment, (view the chaotic_motions.mp4 among the materials [csodafizika]). To study the fractals connected with chaotic motion the use of the phase space is unavoidable. Though it is not more difficult than using the time-dependent representations, students find it more intricate and hardly understandable; therefore, we offer some activities to strengthen its conceptual basis and to illustrate it in practice. The construction of the phase space is illustrated by the run of the file entitled phase_space.html found among the downloadable materials [csodafizika]. The attracting set of the phase space, towards which the trajectories are heading on the long run is called an attractor. The attractor of chaotic systems is of fractal nature that was shown detailedly with the example of Duffing oscillator in another of our papers [Csernovszky, Nagy, Tasnádi (2019)].
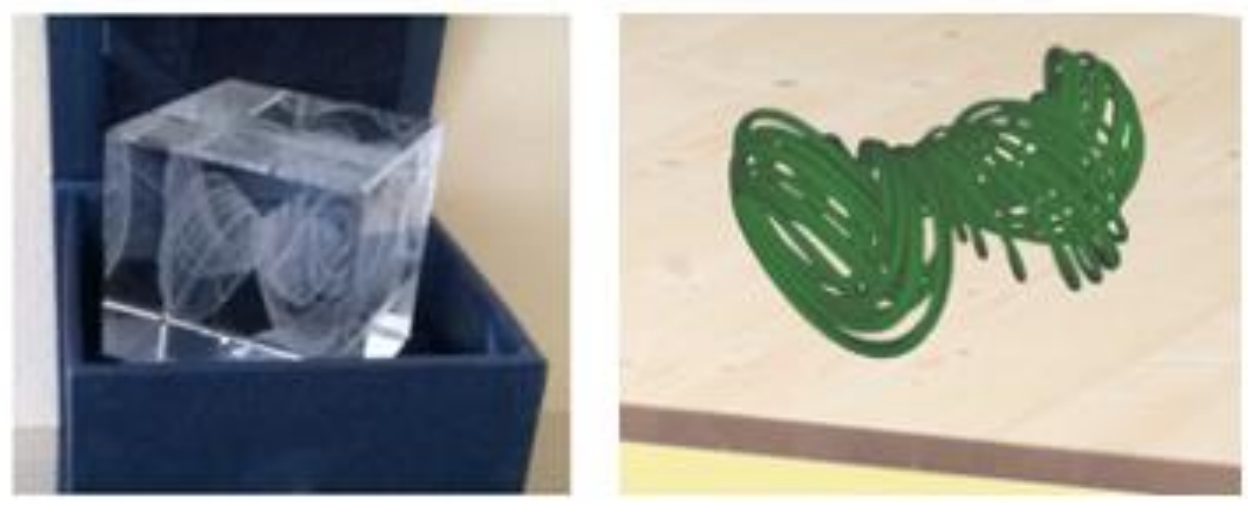

Figure 6. Our Zeeman attractor in glass and its 3D-printed realization.

Finally some words about the visualisation technics. Human beings are visual creatures in nature therefore every aid and procedure that is a visible demonstration of an abstract idea is of great value from a didactic point of view. The images of the $2 \mathrm{D}$ projection of the strange attractors are very well known and common; however, their 3D visualizations give a real sensation for the human brain, so they are much more suitable for capturing the students'interest. Glass engraving, also called laser engraving, is a method for the preparation and presentation of 3D images materialized inside of a transparent solid such as glass or 
crystals which were made about real objects. The pictures in Figure 6 show some illustration of the 3D realizations of our Zeeman-attractor [Nagy, Tasnádi (2019)].

\section{Summary}

The teaching material presented was suitable to help students learning independently the basic concepts of fractals. The material is a versatile summary of the appearance of the fractals in various fields of sciences and it is freely available on the internet. This material fits the curriculum of an optional course "Concepts of Modern Physics" and also that of the postgraduate course of ELTE teacher training. Our experiences show that the material facilitates a deeper understanding of the Newtonian mechanics for students.

\section{Acknowledgements}

This research is supported by EFOP-3.6.1-16-2016-00006 "The development and enhancement of the research potential at John von Neumann University"project. The Project is supported by the Hungarian Government and co-financed by the European Social Fund. This study was funded by the Content Pedagogy Research Program of the Hungarian Academy of Sciences.

\section{References}

authenticationinart.org/pdf/literature/Richard-P.-TaylorOrder-in-Pollocks-Chaos.pdf

Csernovszky, Z., Nagy, P., Tasnádi, P. (2019). Investigation of chaos in the absence of programming skill. In the process of publication in Canadian Journal of Physics (https://www.nrcresearchpress.com/doi/abs/10.1139/cjp-2019-0466\#.XinM_yNCe00)

csodafizika.hu/fractals.zip

fiztan.phd.elte.hu/kutcsop/munkacsoportok/modern/Duffing_DS/Duffingoszcillator_DS.pdf

http://fractalfoundation.org/OFC/OFC-10-4.html

Mandelbrot, Benoit B. (1982). The fractal geometry of nature, Freeman, New York

Nagy, P., Tasnádi, P. (2014). Zeeman catastrophe machines as a toolkit for teaching chaos, European Journal of Physics 35 Paper: 015018, 22 p. 2014.

Nagy, P., Tasnádi, P. (2019). Visualization of chaotic attractors in 3D as motivating tool for introductory physics course, IOP J. Phys.: Conf. Ser.1286 012028, 2019

Tamás Tél, Márton Gruiz: Chatic Dynamics, Cambridge University Press, 2006 